\title{
CALIDAD DEL SERVICIO DE ATENCIÓN, CUMPLIMIENTO DE PROTO- COLOS Y MANEJO DE NORMAS DE BIOSEGURIDAD EN LAS UNIDADES DE CUIDADOS INTENSIVOS
}

\author{
QUALITY OF CARE, COMPLIANCE WITH PROTOCOLS AND MANAGE- \\ MENT OF BIOSAFETY STANDARDS IN INTENSIVE CARE UNITS
}

\section{Marco Antonio Vera Núñez ${ }^{1}$ Mónica Estefanía Ramón Tigse ${ }^{2}$}

1.* Universidad Estatal de Milagro, Ecuador. Email: veranunezm@gmail.com (D) https://orcid.org/0000-0003-0326-5832

2. Hospital de Especialidades Eugenio Espejo, Ecuador. Email: monyrt23@hotmail.com (DD https://orcid.org/0000-0002-8305-253x

Como Citar: Vera Núñez , M. A., \& Ramón Tigse, M. E. (2021). Calidad del servicio de atención, cumplimiento de protocolos y manejo de normas de bioseguridad en las unidades de cuidados intensivos. Revista Publicando, 8(29), 45-53. https://doi.org/10.51528/rp.vol8.id2170

\section{RESUMEN:}

Al hablar de la salud pública se enfatiza constantemente en la seguridad de los usuarios y la calidad de la asistencia médica ofrecida; en el marco de la medicina intensiva se atienden pacientes con enfermedades de naturaleza potencialmente letales, incluyendo aquellos con condiciones altamente inestables. Con el objetivo de indagar sobre la calidad del servicio a la población, factores de riesgos, cumplimiento de protocolos y manejo de normas de bioseguridad en las unidades de cuidados intensivos se realizó una revisión descriptiva de la producción científica durante el último quinquenio. Los estudios revelaron numerosos temas respecto a las infecciones nosocomiales, encontrando en menor medida artículos sobre aspectos éticos, cumplimiento de normativas y del entorno laboral, un importante porcentaje de los estudios insisten en el apoyo al paciente y sus familiares, siendo factores de salud pública de elevada preocupación social.

\section{PALABRAS Clave:}

Salud pública, calidad del servicio, bioseguridad, protocolos, normas.

\section{ABSRTACT:}

When speaking of public health, the safety of users and the quality of the medical assistance offered are constantly emphasized. In the framework of intensive medicine, patients with diseases of a potentially lethal nature are treated, including those with highly unstable conditions. In order to investigate the quality of the service to the population, risk factors, compliance with protocols and management of biosafety standards in intensive care rooms, a descriptive review of scientific production was carried out during the last five years. The studies revealed numerous topics regarding nosocomial infections, finding to a lesser extent articles on ethical aspects, compliance with regulations and the work environment, a significant percentage of the studies insist on supporting the patient and their families, being public health factors of high social concern.

\section{KEYWORDS:}

Public health, quality of service, biosafety, protocols, standards. 
궁 P U B L I C A N D O

$\begin{array}{lllllllllll}\text { I S S N } 13 & 3 & 9 & 0 & -9 & 3 & 0 & 4\end{array}$

\section{INTRODUCCIÓN}

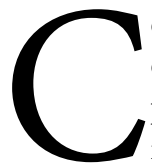

omo es conocido en la atención médica que se ofrece a la ciudadanía no interviene una sola persona, en el sector de la salud se labora generalmente en equipo y es allí donde la calidad del servicio depende de la excelencia del trabajo conjunto y se considera un factor clave en la diferenciación y en la percepción de los usuarios (Amaro, 2019; Romero, Contreras y Cantú, 2019). El criterio de los pacientes sobre la prestación de la salud pública queda determinado definitivamente por el nivel de calidad de las instituciones, así como, la preparación y el servicio que reciben de los profesionales de la salud (Torres et al., 2020). Para evaluar la calidad de los servicios de salud se han referido diversos indicadores de efectividad, aunque se reconoce la complejidad de la misma (Henao, Giraldo y Yepes, 2017).

En la salud, la seguridad de los usuarios queda definida como el descenso al mínimo admisible del riesgo de daño innecesario coligado a la atención (Felippe, Machado y Martins, 2020), y se logra a partir de la planeación del conjunto de procesos que las instituciones ejecutan en todos sus niveles (Mucito y Sánchez, 2020). Por lo tanto, este concepto lleva implícito el contexto en el cual el cuidado y la adecuada atención son dispensados; en el compromiso de ofrecer mejor salud a la ciudadanía se deberá siempre reducir el daño como efecto sobre la persona, sea físico o psicosocial, lo cual se resume en restringir al máximo las enfermedades, sufrimiento, incapacidad o disfunción de los usuarios, así como mejorar la calidad de vida de los pacientes, cuidadores y sus familias (Pérez, López y Torres, 2020).

Dentro de la salud pública, la práctica del cuidado intensivo ha tenido cambios sustanciales a favor del usuario con el pasar del tiempo, actualmente se viene observando un constante progreso para el tratamiento de las enfermedades y/o padecimientos potencialmente letales, es allí donde el cumplimiento de los protocolos y la detección de los factores de riesgo serán elementos claves para reducir las complicaciones del usuario y gracias al propio desarrollo tecnológico se puede diagnosticar y monitorizar a los mismos de una forma rápida y adecuada.

Las infecciones asociadas a la atención en salud en los países en vías de desarrollo, es de 2 a 20 veces mayor que en los países desarrollados, siendo el incidente desfavorable que aparece con mayor frecuencia durante la prestación de atención sanitaria, reflejando las estadísticas a escala global que alrededor de 1,4 millones de personas contraen infecciones en los hospitales (OMS, 2020), además este crucial tema no ha sido resuelto como problema de salud pública en Ecuador, presentándose elevados índices en el servicio de Terapia Intensiva (González et al. 2019).

Como problema de salud pública las infecciones contraídas en las unidades de cuidados intensivos se mantienen como uno de los inconvenientes significativos que preocupan al personal de salud y a la población en general, generando situaciones de estrés dado el entorno y el abordaje biomédico que allí se practica (Ruiz, Consuegra y Ruiz, 2018). Respecto a este planteamiento, el estudio se ha fijado el propósito de analizar en el estado del arte, la calidad del servicio prestado a la población, los factores de riesgos, el cumplimiento de protocolos y el manejo de normas de bioseguridad en las unidades de cuidados intensivos.

\section{METODOLOGÍA}

Se realizó un artículo de revisión de tipo exploratorio-descriptivo, empleando el método de síntesis del conocimiento para profundizar el tema en cuestión (Peters et al., 2015), en el cual se consultaron fuentes primarias y secundarias de información a través de las bases de datos Scopus, Medline, Pubmed y Redalyc. Se elaboró una estrategia de búsqueda y selección de los artículos, en el cual quedaron fichados los estudios de mayor relevancia. En la estrategia se plasmaron las siguientes preguntas que sirvieron de orientación del trabajo: ¿Qué aspectos comprenden la calidad del servicio en las salas de cuidados intensivos? ¿Cuáles son los protocolos y normas de bioseguridad a cumplir en las salas de cuidados intensivos? ¿Qué percepción tiene sobre los usuarios brindar un mejor servicio?

Dentro de la literatura consultada se tuvo en cuenta aquellas publicaciones de los últimos cinco años en las revistas científicas, donde el sujeto de estudio quedara relacionado con la calidad del servicio, riesgos, bioseguridad y cuidados intensivos; de los artículos seleccionados se acopió toda la información bibliográfica la cual fue procesada empleando el gestor bibliográfico "Mendeley".

\section{RESULTADOS}

A escala social es permanente la preocupación por la reducción de la morbimortalidad, la mejoría de la calidad de vida y la capacidad funcional de los pacientes que requieren un tratamiento sofisticado (Puente, 2018), es por ello, que en las UCI se requiere de una labor constante entre los profesionales de la salud que como equipo multidisciplinario trabajan coordinada y sistémicamente para la estabilización de los usuarios (Mera et al., 2019).

Es además, una gran preocupación las conocidas infecciones asociadas a la atención en salud (IAAS), estas pueden afligir a los usuarios en cualquier tipo de entorno donde reciben la atención sanitaria (Coello, 2017; González et al., 
2019), ya sean, adquiridas en la institución hospitalaria o las surgidas también después de haber recibido el alta, como plantea la Organización Mundial de la Salud, hasta en ese momento han aparecido complicaciones, tales como: infecciones hospitalarias, caídas, paro cardio-respiratorio, fallecimiento, entre otros (Gotur et al., 2018). En las UCI el inconveniente se subscribe en estar al tanto de los factores que circundan al paciente crítico, en saber programar la atención, cumplir los protocolos y las directrices para otorgarle una mejor calidad de atención (Espinel et al., 2020; Quintana y Tinajero, 2020).

\section{LOS FACTORES DE RIESGO Y TRANS- MISIÓN DE LAS IAAS}

Es reconocida la existencia de marcadores de riesgo en esta instancia, como las características del paciente que no son modificables (sexo y edad) e influyen en la aparición de IAAS (Alvarado, 2020). Estos factores de riesgo incrementan las probabilidades de contraer un problema de salud o una enfermedad, representando un potencial riesgo sanitario para algunos individuos (Romo, 2020).

La literatura científica muestra internacionalmente un gran número de factores de riesgo relacionados con el desarrollo de IAAS como pueden ser: severidad de la enfermedad de base (Galicia et al., 2017), instrumental y técnicas invasivas (Carmen et al., 2017), terapia antimicrobiana (Ozuna et al., 2019), la reducción de la inmunidad y la variedad de procedimientos médicos (González et al., 2019); estos factores predisponen vías de infección, como pueden ser, la transmisión de microorganismos farmacorresistentes en los individuos hospitalizados, influenciado también por prácticas deficientes de control de infecciones que definitivamente dejan abierto el camino para la transmisión de las IAAS (Cruz, 2020).

Ante un desbalance de la tríada ecológica (microorganismos, huésped y medio ambiente), se inician los diversos modos de transmisión de las IAAS; estos patógenos hospitalarios pueden ser transmitidos por los propios trabajadores de la salud y el instrumental médico contaminado, todo lo que, incrementa los riesgos de los usuarios que ingresan en la UCI (Barahona et al., 2019). Ha quedado demostrado en algunos países en vías de desarrollo que más del 25\% de pacientes ingresados pueden quedar afectados por las IAAS (González et al., 2019).

Tabla 1. Resumen de estudios sobre calidad del servicio de atención en la UCI.

\begin{tabular}{lllll}
\hline País & Caso de estudio & Objetivo & Resultados & $\begin{array}{l}\text { Fuente bibli- } \\
\text { ográfica }\end{array}$ \\
\hline Argentina & $\begin{array}{l}\text { Recomendaciones para la } \\
\text { gestión de recursos en las } \\
\text { UCI durante la pandemia. }\end{array}$ & $\begin{array}{l}\text { Proporcionar recomen- } \\
\text { daciones basadas en la } \\
\text { evidencia para estimar } \\
\text { los recursos }\end{array}$ & $\begin{array}{l}\text { El modelo permitió predecir los } \\
\text { recursos necesarios para proporcio- } \\
\text { nar cuidados críticos }\end{array}$ & $\begin{array}{l}\text { (Arias et al., } \\
2020)\end{array}$ \\
Ecuador & $\begin{array}{l}\text { Cuidados asistenciales en } \\
\text { pacientes ingresados en }\end{array}$ & $\begin{array}{l}\text { Mantener la superviven- } \\
\text { cia de los pacientes en } \\
\text { estado crítico }\end{array}$ & $\begin{array}{l}\text { Los malos protocolos de manejos } \\
\text { por el personal pueden causar el } \\
\text { agravamiento de pacientes críticos }\end{array}$ & (Mera et al., \\
& UCI & $\begin{array}{l}\text { Comprender la influ- } \\
\text { encia del proyecto en la }\end{array}$ & $\begin{array}{l}\text { La intervención centrada en el paci- } \\
\text { ente facilita la atención e impacta al }\end{array}$ & (Neville et al., \\
EE.UU. & Mejora de la atención al
\end{tabular}

España Prevención de la Bacteriemia relacionada con catéter venoso central en la UCI.

Cuba
Sistemas de notificación de eventos adversos en UCI. que permiten una vigilancia remota eficaz.

Demostrar la importancia de una adecuada práctica clínica y describir los protocolos de prevención basados en la evidencia.

Sistematizar el conocimiento sobre sistemas de notificación de eventos adversos en UCI. atención al paciente puede beneficiarse de dichos sistemas.

La alta incidencia de bacteriemias nosocomiales y su mortalidad, hace necesaria la implantación de protocolos de prevención.

Los sistemas de notificación son una herramienta importante para aumentar la calidad de los servicios.
(Cuezva, 2019)

(Mora et al., 2020) 


\begin{tabular}{|c|c|c|c|c|}
\hline País & Caso de estudio & Objetivo & Resultados & $\begin{array}{l}\text { Fuente bibli- } \\
\text { ográfica }\end{array}$ \\
\hline Colombia & $\begin{array}{l}\text { Estrategias de afronta- } \\
\text { miento en familiares de } \\
\text { pacientes críticos. }\end{array}$ & $\begin{array}{l}\text { Describir las estrategias } \\
\text { de afrontamiento de los } \\
\text { familiares de pacientes } \\
\text { críticos. }\end{array}$ & $\begin{array}{l}\text { Se deben priorizar el trato hu- } \\
\text { manizado y una comunicación } \\
\text { acorde con las necesidades de los } \\
\text { familiares. }\end{array}$ & $\begin{array}{l}\text { (Ochoa et al., } \\
2020)\end{array}$ \\
\hline Perú & $\begin{array}{l}\text { Efectividad de los cuidados } \\
\text { paliativos en cuidados } \\
\text { intensivos. }\end{array}$ & $\begin{array}{l}\text { Revisar y sistematizar la } \\
\text { efectividad de los cuida- } \\
\text { dos paliativos en la UCI. }\end{array}$ & $\begin{array}{l}\text { Los cuidados paliativos en la UCI } \\
\text { son efectivos ya que proporcionan } \\
\text { beneficio al paciente y a la familia. }\end{array}$ & $\begin{array}{l}\text { (Ríos y Torres, } \\
\text { 2019) }\end{array}$ \\
\hline
\end{tabular}

Nota: Elaborado por autores.

Sobre las IAAS se afirma que no existe ningún país que haya resuelto esta problemática de salud pública, muy relacionada con la bioseguridad y la calidad de atención a los usuarios, la cual ha sido considerada en varios trabajos investigativos (Tabla 1), ya que se observan como uno de los incidentes adversos con mayor frecuencia en la prestación de atención sanitaria.

Se ha venido realizando un gran esfuerzo en los países para disminuir estos factores de riesgo en los pacientes atendidos en las UCI, con ello se debe contraer la aparición de las IAAS, sin embargo, será necesario identificarlos como paso previo para la elaboración de estrategias de su prevención, aunque la realidad, a decir de la Organización Panamericana de la Salud, es bajo el porcentaje de los hospitales que poseen un activo y acreditado comité de infecciones. El negativo impacto que incitan las IAAS es un indicador para la búsqueda de estrategias que aminoren esta problemática que también afecta al personal de los hospitales.

Por ello se expresan algunas estrategias preventivas para reducir las IAAS, en este caso:

- Identificar cuáles son las infecciones más frecuentes.

- Cuáles son los microorganismos.

- Análisis de los posibles factores o causas de aparición.

- Elaboración de las normas.

- Capacitación al personal.

- Supervisión de las labores realizadas.

- Evaluación del cumplimiento de las normativas.

- Evaluación del impacto de las intervenciones.

A estos aspectos relacionados se les agrega la necesaria atención de los protocolos para el manejo racional de antibióticos y el cumplimiento de las medidas universales de bioseguridad; ante esta preocupación los directivos y profesionales que trabajan en hospitales deberán estar al tanto e implementar programas de preventivos para la vigilancia y el control de las IAAS al ser un indicador de calidad altamente sensible en la prestación de servicios de salud (Lara et al., 2020).

\section{CALIDAD DEL SERVICIO}

No basta con mejorar el acceso a la salud, es importante garantizarles a las personas una atención de calidad y para ello se debe proveer un excelente servicio de salud, siendo esencial para alcanzar la cobertura sanitaria universal (Kruk et al., 2017). Según recomendaciones de la OMS se ha solicitado a las autoridades sanitarias a emplazar políticas nacionales que mejoren la calidad de los servicios de salud, siendo deseable combinarlas de forma sistemática y coordinada con los procesos de mejora de la atención en todo el sistema (Shamsuzzoha et al., 2018; Figueroa, 2020).

Ese compromiso de elevar la calidad del servicio, diseñado por las estructuras de gobernanza en los diferentes niveles de dirección, para propiciar un cambio en la cultura organizacional del sistema de salud y que impulse el desvelo por la calidad de la atención para los usuarios, es fundamental en la medicina intensiva, la que se ocupa del paciente en estado crítico, quienes alcanzan un nivel de gravedad que puede representar una potencial amenaza para su vida (Jacobsen et al., 2019), esos pacientes susceptibles de recuperación tienen como característica que requieren un área más tecnificada, con asistencia y cuidados continuos (Ochoa, 2017).

Con el afán de asegurar la calidad de la asistencia médica y la seguridad del paciente se plantea será siempre necesario profesionales capacitados en cada área de la salud (Lopes y D'Innocenzo, 2017); el elevado potencial de letalidad de los padecimientos de la población que ingresa a la UCI, el uso de procedimientos invasivos y el tratamiento sofisticado que requieren resalta el trabajo multidisciplinario del equipo que allí labora, por ende, todos se integrarán en un sistema que brinde la adecuada calidad en la atención ofrecida (Figura 1). 


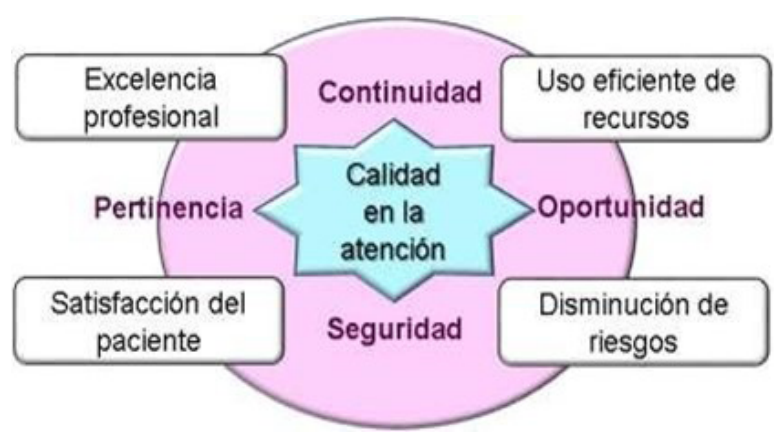

Figura 1: Integración de los factores que inciden en la calidad de la atención ofrecida. Elaborado por autores.

\section{CUMPLIMIENTO DE PROTOCOLOS}

Para el logro de la calidad de atención clínica, los profesionales de la salud asumen decisiones, gran parte de ellas aprendidas durante su preparación en el ciclo formativo, luego reciben múltiples cursos y capacitaciones, que unidas a la transmisión de experiencias de los expertos en su trabajo les permite integrar y fortalecer sus habilidades y competencias; también cuentan con protocolos que recogen el conjunto de actividades a ejecutar por parte del personal que labora en la institución de salud, tratan sobre las actividades asistenciales o atienden problemas específicos del área.

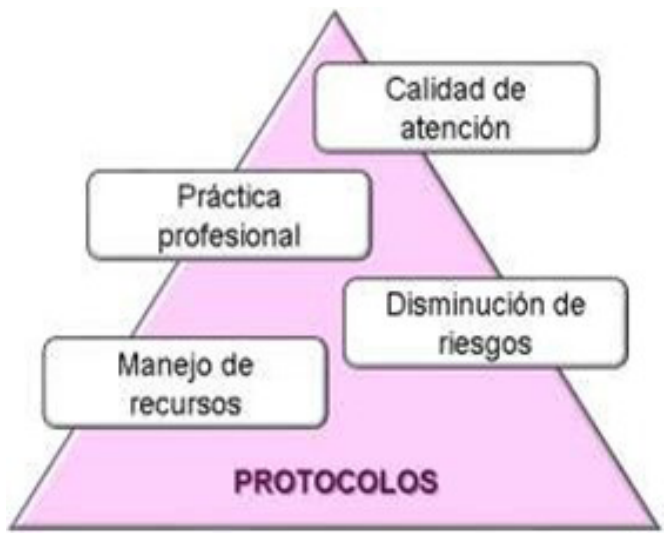

Figura 2: Elementos de los protocolos para el logro de la calidad de atención. Elaborado por autores.

El uso de protocolos como guías de práctica clínica mejoran la calidad en la asistencia sanitaria, conducen a un mejor manejo de recursos, integran procedimientos necesarios para la atención sanitaria, contribuyen a la disminución de riesgos, disminuye las informalidades en la práctica clínica y unifica los criterios de actuación entre los profesionales, por lo tanto, ayuda en la asistencia sanitaria y con ello es mejor la calidad de la atención.

\section{MANEJO DE NORMAS DE BIOSEGURI- DAD}

Como parte de los lineamientos operativos existen las normas de bioseguridad en las instituciones de salud, las cuales cumplen el objetivo de prevenir y/o advertir accidentes laborales y reducir el riesgo de aparición de infecciones cruzadas causadas por el insuficiente empleo de equipos de protección personal, la escasa disponibilidad de insumos, el mal manejo de desechos cortopunzantes, entre otros ( $\mathrm{Zu}-$ ñiga, 2019).

Los principios de la bioseguridad aplican en cualquier circunstancia laboral, siendo muy relevantes en el área hospitalaria, fundamentalmente por estar en contacto directo con individuos enfermos, situación que puede desatar un determinado

contagio, por lo que la consiguiente toma de las medidas adecuadas entre los profesionales de la salud será un factor clave para evitar la transmisión de las enfermedades infecciosas.

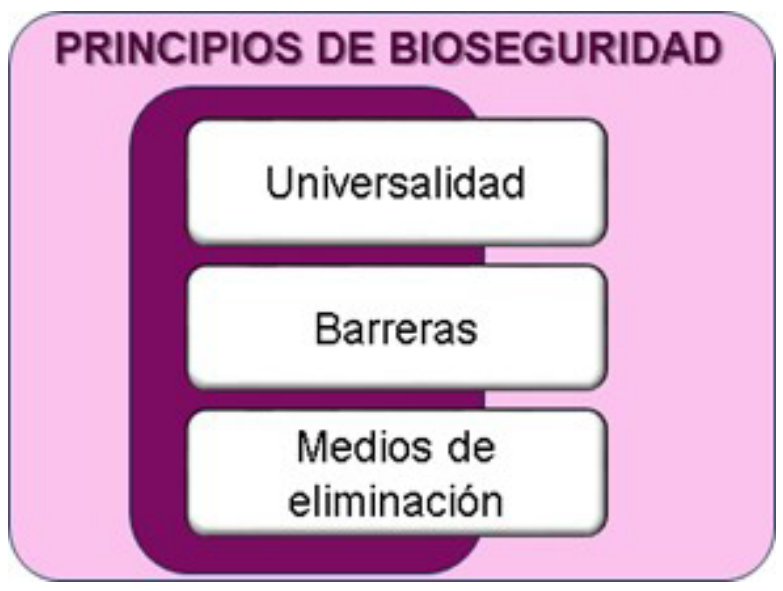

Figura 3: Principios de bioseguridad. Elaborado por autores.

- Universalidad: cobra sentido cuando se deduce la aplicación de las medidas preventivas que involucran las prácticas y alcanzan a todos los individuos, se registre o no una patología.

- Barreras de Contención: a través de estas se evita la exposición directa al riesgo, con el uso de elementos de protección primaria, equipamiento, materiales y 
procedimientos apropiados.

- Manejo de Residuos: con ello se revelan los dispositivos propicios y la forma correcta para la eliminación de todo material contaminado.

Aspectos que se incluyen dentro de las normas de bioseguridad (Ministerio de Salud Pública del Ecuador, 2016):

- Lavarse las manos antes y después de realizar cualquier procedimiento.

- Utilizar guantes, mascarilla, delantales y gafas de protección para no exponerse a fuentes potenciales de infección.

- Evadir el contacto de la piel y mucosas con la sangre y líquidos corporales procedentes del paciente.

- Poner exclusiva atención en la manipulación de los utensilios de trabajo.

- Estar concentrado en las actividades.

- Retirar los implementos cortopunzantes.

- Ante la presencia de piel no intacta por lesiones, se debe evitar el contacto directo con pacientes que estén eliminando líquidos corporales.

La aplicación de un plan de bioseguridad requeriría, necesariamente, de una organización institucional que vigile el cumplimiento de las normativas establecidas. De esta forma, se recomienda que cada institución constituya un Comité Institucional de Bioseguridad (CIB) que se encargue de formular las políticas y prácticas internas en materia de bioseguridad, examinar los protocolos de investigación, evaluación de riesgos, vigilancia y solución de controversias (Fondecyt, 2018).

\section{CONCLUSIONES}

Los estudios científicos demostraron que, para maximizar la calidad del servicio en la UCI, el cumplimiento de protocolos y el manejo de normas de bioseguridad en las salas de cuidados intensivos, serán vitales en la disminución de la aparición de las IASS, a la vez que, redundarán en la mejora de la calidad de la práctica clínica. De igual manera permitirá establecer directrices que ayuden a sistematizar los procesos y a minimizar las variaciones inadecuadas en el actuar médico y de enfermería.

El cumplimiento de los protocolos establecidos, priorizar la distribución de los recursos sanitarios y el seguimiento de las normas de bioseguridad y su control, hará que sea más eficiente el uso de los mismos y que la calidad del servicio prestado sea excelente. Los estudios revelaron numerosos temas respecto a las IASS, encontrando en menor medida artículos sobre aspectos éticos, cumplimiento de normativas y del entorno laboral, un importante porcentaje de los estudios insisten en el apoyo al paciente y sus familiares, siendo factores de salud pública de elevada preocupación social.

\section{CONFLICTO DE INTERESES}

Los autores declaran no tienen ningún conflicto de intereses. 


\section{지 P U B L I C A N D O \\ I S S N $133900-93304$}

\section{REFERENCIAS}

Alvarado, A. K. (2020). Características clínicas del paciente geriátrico que desarrolló neumonía intrahospitalaria en el Hospital Santa Rosa 2012-2019. Retrieved from http://repositorio.unfv.edu.pe/handle/ $\mathrm{UNFV} / 4215$

Amaro, M. del C. (2019). La ética de la gestión, uno de los factores determinantes de la calidad de los servicios de salud. Revista de Información Para La Dirección En Salud (INFODIR)., 29(3), 1-10. Retrieved from http://revinfodir.sld.cu/index.php/infodir/article/ view/615/878

Arias, M. D., Mandich, V., Mosciaro, M., Ratto, M. E., Fresco, J. C., \& Boada, N. (2020). Recomendaciones para la gestión de recursos en las unidades de cuidados intensivos durante la pandemia de COVID-19. MEDICINA (Buenos Aires), 80(Supl III), 67-76. Retrieved from http://www.medicinabuenosaires. com/revistas/vol80- 20/destacado/revision_7224. pdf

Barahona, N., Martína, M., \& de Moya, Y. (2019). Importancia de la vigilancia epidemiológica en el control de las infecciones asociadas a la atención en salud. biociencias, 14(1), 65-81. Retrieved from https://dialnet.unirioja.es/servlet/articulo?codigo $=7380540$

Carmen, S. P., Pérez, R. V, \& Exebio, I. D. (2017). Conocimiento de las enfermeras sobre Infecciones Intrahospitalarias en el Hospital Regional Lambayeque, Chiclayo, Perú. ACC CIETNA: Revista de la Escuela de Enfermería, 4(2), 39-49. Retrieved from http:// revistas.usat.edu.pe/index.php/cietna/article/ view/18

Coello, C. (2017). Ecuador con alta tasa de mortalidad por infecciones nosocomiales. Retrieved from https:// www.edicionmedica.ec/secciones/salud- publica/ ecuador-con-la-mayor-tasa-de-mortalidad-por-infecciones- nosocomiales-89901

Cruz, F. (2020). Impacto de la colonización de pacientes en el desarrollo de infecciones asociadas a la atención de la salud (Doctoral dissertation, Universidad Autónoma de Nuevo León). Retrieved from http:// eprints.uanl.mx/19882/

Cuezva, I. (2019). Prevención de la bacteriemia relacionada con catéter venoso central en las unidades de cuidados intensivos. Retrieved from https://uvadoc.uva. es/handle/10324/39075

Espinel, D. E., Nuñez, M. M., \& Ordoñez, V. E. (2020). Complicaciones de la ventilación mecánica invasiva en cuidados intensivos: una scoping review. Retrieved from https://repository.ucc.edu.co/handle/20.500.12494/20213

Felippe, V., Machado, S. da C., \& Martins, M. (2020). Seguridad del paciente en el cuidado hospitalario: una revisión sobre la perspectiva del paciente. Cad. Saúde Pública 2020;, 36(12). https://doi.org/10.1590/0102-311X00223019

Figueroa, L. (2020). Estrategias para la prevención y control de las infecciones asociadas a la atención sanitaria. Revista del Hospital" Dr. Emilio Ferreyra", 1(2), e35-e44. Retrieved from http://revista.deiferreyra. com/index.php/RHEF/article/view/24

Fondecyt. (2018). Manual de Normas de Bioseguridad y Riesgos Asociados. Retrieved from https://www. conicyt.cl/fondecyt/files/2018/06/Manual_Bioseguridad_junio_2018.pdf

Galicia, I. S., Cámara, K. B., \& Castañeda, M. D. (2017). La Frecuencia de IAAS en UCIN del Hospital de Playa del Carmen de enero a diciembre del 2016. Revista Salud y Bienestar social [ISSN: 2448-7767], 1(2), 33-46. Retrieved from https://www.revista. enfermeria.uady.mx/ojs/index.php/Salud/article/ view/22

González, J. A., Noriega, D. D., Escariz, L. I., \& Mederos, K. (2019). Incidencia de factores de riesgo de infecciones, asociadas a la atención en salud en pacientes críticos. Revista San Gregorio, (31). Retrieved from http://revista.sangregorio.edu.ec/index.php/REVISTASANGREGORIO/article/v iew/862/11-JONAT2

Gotur, D., Masud, F., Paranilam, J., \& Zimmerman, J. (2018). Analysis of Rothman Index Data to Predict Postdischarge Adverse Events in a Medical Intensive Care Unit. J Intensive Care Med, 2, 1-5. https://doi. org/10.1177/0885066618770128

Henao, D., Giraldo, A., \& Yepes, C. (2017). Instrumentos para evaluar la calidad percibida por los usuarios en los servicios de salud. Revista Gerencia y Políticas de Salud, 2018, 17(34). https://doi.org/https://doi. org/10.11144/Javeriana.rgps17-34.iecp 
Jacobsen, K. S., Ott, P., \& Eriksen, P. L. (2019). Spontaneous bacterial peritonitis a shift in bacteria and resistance pattern. Scandinavian Journal of Gastroenterology, 1-3. https://doi.org/10.1080/00365521.2019. 1697896

Kruk, M., Kelley, E., Syed, S., Tarp, F., Addison, T., \& Akachi, Y. (2017). Measuring quality of health-care services: what is known and where are the gaps? Bull World Health Organ. 2017 Jun 1;95(6):389-389A. Retrieved from http://dx.doi.org/10.2471/BLT.17.195099 pmid: 28603302

Lara, G., Ariosa, C., Borroto, V., Puerta, Á., Ortiz, R., \& Villalobos, C. (2020). Ozono como método de desinfección del ambiente hospitalario. Acta Médica Costarricense, 62(2), 72-78. Retrieved from https://www.scielo.sa.cr/scielo.php?pid=S0001$60022020000200072 \&$ script $=$ sci_arttext

Lopes, M., \& D’Innocenzo, M. (2017). Eventos adversos relacionados con las prácticas asistenciales: una revisión integradora. Enfermería Global, (47), 605620. Retrieved from http://scielo.isciii.es/pdf/eg/ v16n47/1695-6141-eg-16-47-00605.pdf

Mera, Á. R., Carranza, L. H., Campuzano, P. G., Intriago, O. L., Falconí, M. H., \& Alvear, G. A. (2019). Cuidados asistenciales en pacientes ingresados en UCI. RECIAMUC, 3(3), 1142-1155. Retrieved from https:// reciamuc.com/index.php/RECIAMUC/article/ view/321

Ministerio de Salud Pública del Ecuador. (2016). Bioseguridad para los establecimientos de salud. Manual. Ministerio de Salud Publica, 227.

Mora, Y., Bosi de Souza, T., Torres, J., \& Alfredo, E. (2020). Sistemas de notificación de eventos adversos en unidades de cuidados intensivos para gestión de riesgo. Revista Cubana de Enfermería, 36(3). Retrieved from https://www.medigraphic.com/pdfs/ revcubenf/cnf-2020/cnf203o.pdf

Mucito, E., \& Sánchez, F. V. (2020). Tecnovigilancia en los laboratorios clínicos: una herramienta para la seguridad del paciente. Rev CONAMED, 25(4), 174181. https://doi.org/10.35366/97337

Neville, T., Agarwal, N., Swinton, M., Phung, P., Xu, X., Kao, Y., \& Pavlish, C. (2019). Mejora de la atención al final de la vida en la unidad de cuidados intensivos: experiencias de los médicos con el Proyecto 3 De- seos. Revista de Medicina Paliativa , 22 (12), 15611567. Retrieved from https://doi.org/10.1089/ jpm.2019.0135

Ochoa, K., López, C. M., Guzmán, S. Y., Ruíz, D. P., \& Zambrano, S. M. (2020). Estrategias de afrontamiento en familiares de pacientes críticos. Revista Repertorio de Medicina y Cirugía, 1-8. Retrieved from https://revistas.fucsalud.edu.co/index.php/repertorio/article/view/950

Ochoa, M. (2017). Historia y evolución de la medicina crítica: de los cuidados intensivos a la terapia intensiva y cuidados críticos. Acta Colombiana de Cuidado Intensivo, 17(4), 258-268. https://doi.org/10.1016/j. acci.2017.08.006

OMS. (2020). Una atención más limpia es una atención más segura. Retrieved from https://www.who.int/gpsc/ background/es/

Ozuna, M., Vester, L., \& Jiménez, J. (2019). Implementación de medidas preventivas de las Infecciones Asociadas a la Atención de Salud (IAAS) en un departamento de cuidados intensivos pediátricos. Revista Científica en Ciencias de la Salud-ISSN: 2664-2891, 1(2). Retrieved from https://bit.ly/39LjxPp

Pérez, D., López, J., \& Torres, C. (2020). Cuidados paliativos para pacientes con infección por SARS-CoV-2/COVID-19; propuesta de un modelo de atención. Revista Mexicana de Anestesiología, 43(4), 288-295. https://doi.org/10.35366/94942

Peters, M., Godfrey, C., Khalil, H., McInerney, P., Parker, D., \& Soares, C. (2015). Guidance for conducting systematic scoping reviews. Int J Evid Based Healthc. 2015 Sep;13(3):141-6. Doi: 10.1097/ XEB.0000000000000050.

Puente, P. (2018). Influencia de factores modificables en morbimortalidad de pacientes con diabetes mellitus (Doctoral dissertation, Universidad de Guayaquil. Facultad de Ciencias Médicas. Carrera de Medicina). Retrieved from http://repositorio.ug.edu. ec/handle/redug/31339

Quintana, M., \& Tinajero, R. (2020). Investigación en enfermería en unidad de cuidado intensivo del adulto: una revisión de literatura. Horizonte De Enfermería, 30(1), 76-87. https://doi.org/10.7764/Horiz_ Enferm.30.1.76-87. 
Rios, V. H., \& Torres, B. A. (2019). Efectividad de los cuidados paliativos en cuidados intensivos. Retrieved from http://repositorio.uwiener.edu.pe/handle/123456789/2983

Romero, E., Contreras, D., \& Cantú, G. (2019). Calidad de la atención médica desde un punto de vista bioético en un hospital oftalmológico de Ciudad de México. Acta Bioethica, 25(2), 235-242. Retrieved from https://scielo.conicyt.cl/pdf/abioeth/v25n2/1726569X-abioeth-25-2-00235.pdf

Romo, M. D. (2020). Infecciones asociadas a la atención de salud en pacientes ingresados en el hospital San Vicente de Paúl (Master's thesis). Retrieved from http://repositorio.utn.edu.ec/handle/123456789/9800

Ruiz, D., Consuegra, R., \& Ruiz, I. (2018). Factores estresores en pacientes en unidades de cuidado intensivo adulto. Revista de Enfermagem Referência, (16), 63. https://doi.org/10.12707 / RIV17060

Safavi, K. C., Driscoll, W., \& Wiener, J. (2019). Tecnologías de vigilancia remota: lograr el objetivo del paciente adecuado, los datos adecuados y el momento adecuado. Anesth Analg, 129(3), 726-734. https://doi. org/10.1213/ANE.0000000000003948

Shamsuzzoha, S., Sheila, L., Mensah-Abrampah, N., Matthew, N., \& Edward, K. (2018). Mejorar la calidad de la atención de salud en todo el sistema sanitario. Boletín de La Organización Mundial de La Salud 2018;96:799. Doi: Http://Dx.Doi.Org/10.2471/ BLT.18.226266

Torres, R. M., Torres, R., \& Morera, M. (2020). Calidad de la atención médica percibida en el Centro Nacional de Cirugía de Mínimo Acceso. Información Para Directivos de La Salud (INFODIR). 2020;32:E770 Artículo. Retrieved from http://www.revinfodir. sld.cu/index.php/infodir/article/view/770/1101

Zuñiga, J. X. (2019). Cumplimiento de las normas de bioseguridad. Unidad de Cuidados Intensivos. Hospital Luis Vernaza, 2019. Revista Eugenio Espejo, 13(2), 28-41. Retrieved from http://eugenioespejo.unach. edu.ec/index.php/EE/article/view/149/129 